Revue d'histoire de l'Amérique française

Q4. REVUE D.HISTOIRE DE L'AMÉRIQUE FRANÇAISE

\title{
La République du Madawaska et l'Acadie
}

La construction identitaire d'une région néo-brunswickoise au $\mathrm{XX}^{\mathrm{e}}$ siècle

\section{Jacques Paul Couturier}

Volume 56, numéro 2, automne 2002

URI : https://id.erudit.org/iderudit/007315ar

DOI : https://doi.org/10.7202/007315ar

Aller au sommaire du numéro

\section{Éditeur(s)}

Institut d'histoire de l'Amérique française

\section{ISSN}

0035-2357 (imprimé)

1492-1383 (numérique)

Découvrir la revue

\section{Citer cet article}

Couturier, J. P. (2002). La République du Madawaska et l'Acadie : la construction identitaire d'une région néo-brunswickoise $\mathrm{au} \mathrm{xx}^{\mathrm{e}}$ siècle. Revue d'histoire de l'Amérique française, 56(2), 153-184.

https://doi.org/10.7202/007315ar

\section{Résumé de l'article}

Parmi tous les îlots identitaires de la francophonie maritimienne, celui du Madawaska, situé au nord-ouest du Nouveau-Brunswick, affiche haut et fort sa distinction. Dans cette région francophone peuplée par des colons acadiens et canadiens-français, l'identité régionale, dans son incarnation la plus poussée, prend parfois même la forme d'une négation de l'appartenance de la région à l'Acadie contemporaine et la promotion d'un autre type identitaire, le Brayon de la mythique République du Madawaska. Cet article examine le processus de construction identitaire au Madawaska au $\mathrm{XX}^{\mathrm{e}}$ siècle. Il cherche à mettre au jour les représentations identitaires qui prennent forme dans la région et à les mettre en lien avec l'identité acadienne. Entre la promotion des liens du Madawaska à l'Acadie et la mise en valeur des traits régionaux distinctifs, il montre que les représentations identitaires régionales se recomposent au fil du siècle, au fur et à mesure que l'environnement se modifie. 


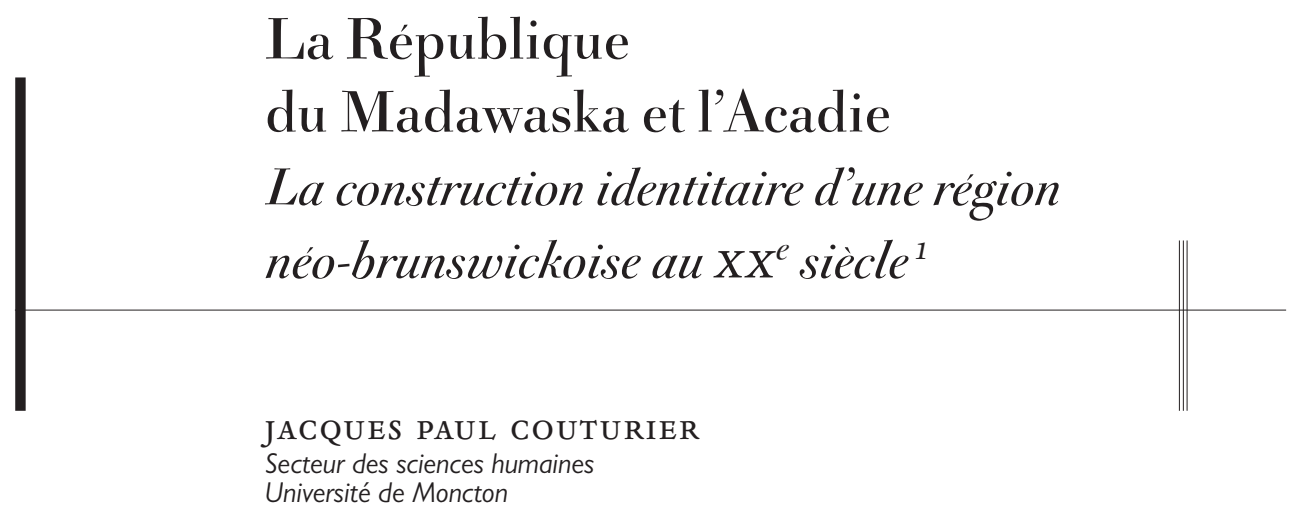

RÉSUMÉ - Parmi tous les îlots identitaires de la francophonie maritimienne, celui du Madawaska, situé au nord-ouest du Nouveau-Brunswick, affiche haut et fort sa distinction. Dans cette région francophone peuplée par des colons acadiens et canadiens-français, l'identité régionale, dans son incarnation la plus poussée, prend parfois même la forme d'une négation de l'appartenance de la région à l'Acadie contemporaine et la promotion d'un autre type identitaire, le Brayon de la mythique République du Madawaska. Cet article examine le processus de construction identitaire au Madawaska au XXe siècle. II cherche à mettre au jour les représentations identitaires qui prennent forme dans la région et à les mettre en lien avec l'identité acadienne. Entre la promotion des liens du Madawaska à l'Acadie et la mise en valeur des traits régionaux distinctifs, il montre que les représentations identitaires régionales se recomposent au fil du siècle, au fur et à mesure que l'environnement se modifie.

ABSTRACT - Of all the francophone islands dotted throughout the Maritimes, none proclaims its distinctiveness more vigorously than Madawaska, in Northwestern New Brunswick. In this francophone region settled by Acadian and French-Canadian colonists, regional identity in its most extreme form is sometimes even expressed as a rejection of the region's ties to contemporary Acadia and the promotion of an alternative identity, the Brayon of the mythical Republic of Madawaska. This article examines the process of identity construction in $20^{\text {th }}$ century Madawaska. It seeks to shed light on the representations of identity that take shape in the region and to understand their relationship to Acadian

1. Cette recherche a pu bénéficier du travail réalisé par mon assistante de recherche, Cindy Harquail, de même que des commentaires de mes collègues Maurice Basque et Nicole Lang ainsi que du cinéaste Rodolphe Caron, et je tiens à les en remercier. 
identity. From the promotion of links between Madawaska and Acadia to the highlighting of distinctive regional characteristics, it demonstrates that the representations of identity were gradually transformed during the century, as the environment changed.

$\mathrm{U}$

N DES TRAITS CARACTÉRISTIQUeS des populations francophones des provinces maritimes, c'est la force et la résilience des identités régionales. De la baie Sainte-Marie (N-É) à la Péninsule acadienne (N-B) en passant par la région Évangéline (IPÉ), les habitants des Maritimes sont avant toute chose de leur "pays", de leur coin de territoire où ils plongent leurs racines. Le phénomène de l'identité régionale est très puissant, en particulier au Nouveau-Brunswick francophone, comme l'illustre avec humour l'auteur Rino Morin Rossignol dans sa pièce Le pique-nique et comme le démontrent plusieurs travaux de recherche. Le géographe Jean-Claude Vernex a ainsi pu écrire, dans les années 1970, qu'il ne fallait pas parler de «la» population francophone du Nouveau-Brunswick, mais plutôt «des » populations francophones du Nouveau-Brunswick ${ }^{3}$. L'identification à une région colore souvent le débat sociopolitique et les rapports entre populations parlant la même langue et partageant une histoire commune. Le cas de l’Université de Moncton est à cet égard particulièrement révélateur. Dès sa fondation en 1963, l'université acadienne est devenue un enjeu du débat entre les régions francophones; elle l'est demeurée par la suite à différentes étapes de sa croissance, en particulier dans la première moitié des années $1970^{4}$.

Parmi tous les îlots identitaires que l'on retrouve au Nouveau-Brunswick, celui du nord-ouest de la province, peuplé par environ 50000 personnes en 1996, dont $89 \%$ sont francophones, affiche le plus ouvertement sa distinction $^{5}$. Celle-ci s'incarne aujourd'hui pour un grand nombre de ces personnes

2. Rino Morin Rossignol, Le pique-nique (Moncton, Perce-Neige, 1982).

3. Jean-Claude Vernex, Les francophones du Nouveau-Brunswick : géographie ethnoculturelle d'un groupe minoritaire (Lille, Atelier de reproduction des thèses de l'Université de Lille, 1978), 1:309. Voir aussi, entre autres, Luc Desjardins, Nationalisme et régionalisme : une analyse spatiale de la cohésion nationale en Acadie du Nouveau-Brunswick, thèse de maîtrise (sciences politiques), Université Laval, 1981, et Patrick Clarke, «Régions et régionalismes en Acadie. Culture, espace, appartenance", Recherches sociographiques, XLI,2 (2000) : 299-365.

4. Sur le sujet, voir Jacques Paul Couturier, Construire un savoir : l'enseignement supérieur au Madawaska, 1946-1972 (Moncton, Éditions d'Acadie, 1999), et Clément Cormier, L'Université de Moncton : historique (Moncton, Centre d'études acadiennes, 1975).

5. Les données sont celles du recensement de 1996 pour la région socio-sanitaire provinciale numéro 4, qui comprend les sous-régions d'Edmundston, Grand-Sault et Saint-Quentin Kedgwick. Cette dernière ne fait toutefois pas partie du Madawaska «historique», dont il sera question dans les pages qui suivent. Sur la délimitation du territoire régional, voir Adrien Bérubé, «La Marévie? Qu'est-ce que c'est?», Revue de la Société historique du Madawaska, VII,2-3 (avril-septembre 
dans l'identification à un territoire, le Madawaska, enclavé entre le Québec, le Maine et le Nouveau-Brunswick anglophone, de même que dans l'appartenance à un groupe francophone issu d'une colonisation mixte - acadienne puis canadienne-française - à la fin du $\mathrm{xvIII}^{\mathrm{e}}$ siècle. Son histoire a été consignée dans un ouvrage publié en 1920, l'Histoire du Madawaska de l'abbé Thomas Albert, seule synthèse d'histoire régionale au Nouveau-Brunswick francophone ${ }^{6}$. L'affirmation de l'identité régionale au Madawaska prend corps depuis 1979 dans une grande fête populaire, la Foire brayonne, où tout un chacun peut renouer avec les sources qui façonnent la personnalité régionale, notamment l'histoire et les traditions culinaires (et plus particulièrement la célèbre galette de sarrasin, la ploye $)^{7}$. Elle débouche sur la valorisation d'une entité imaginaire, la République du Madawaska, le symbole même du fier esprit d'indépendance des habitants du nord-ouest de la province, dotée de son drapeau et d'autres formes de représentations officielles. Dans son incarnation la plus poussée, l'identité régionale prend même parfois la forme d'une négation de l'appartenance à l'Acadie et de l'identité acadienne, à laquelle certains opposent un autre type identitaire, le «Brayon». Territoire, histoire, représentations officielles, identifications pittoresques : le Madawaska dispose dans son bagage d'un bon nombre des éléments symboliques et matériels qui entrent en jeu dans la fabrication des identités nationales ${ }^{8}$.

L'exception identitaire madawaskayenne a souvent été relevée dans les travaux sur l'Acadie, car elle renvoie à un autre débat, celui sur la définition de l'identité acadienne elle-même. Tous les analystes s'empressent en effet de noter que le Madawaska, en raison des sources de son peuplement, de son isolement géographique des autres groupes francophones de la province et des caractéristiques de son milieu naturel, où l'agriculture et l'exploitation forestière ont longtemps dominé, a vu émerger en son sein

1979) : 2-3. Les données du recensement de 1996 sont disponibles en ligne sur le site Internet de Statistique Canada : «Profil statistique - statistiques démographiques pour la Région 4 (Régions socio-sanitaires), Nouveau-Brunswick», www.statcan.ca, page consultée le 10 octobre 2002.

6. Thomas Albert, Histoire du Madawaska, nouvelle édition établie par Adrien Bérubé, Benoît Bérubé et Georgette Desjardins (LaSalle/Edmundston, Hurtubise HMH/Société historique du Madawaska, 1982). Sur la question du peuplement, voir notamment 99-101 (nouvelle pagination). Voir aussi Béatrice Craig, «Immigrants in a Frontier Community : Madawaska 1785-1850», Histoire sociale/Social History, XIX,38 (novembre 1986) : 277-297.

7. Voir Oneil Clavet, «The Republic of Madawaska», Atlantic Advocate, 71,10 (1981) : 46-49; Don Cayo, «Republic of Madawaska : A Modern Myth Rooted in New Brunswick History », Canadian Geographic (janvier-février 1993) : 50-59; et Will Ferguson, «Renegade Republic», Telegraph-Journal, Saint-Jean, 23 janvier 1999.

8. Voir Anne-Marie Thiesse, La création des identités nationales - Europe XVIII-XX $X^{e}$ siècle (Paris, Seuil, 1999), notamment 13-14. 
une collectivité aux traits particuliers. «Les habitants de cette région, constate Luc Desjardins dans un mémoire de maîtrise en sciences politiques sur le régionalisme, ont développé [...] une personnalité collective originale.» «Dans le Nord-Ouest, note pour sa part le sociologue Marc Johnson, on retrouve une affirmation identitaire régionale particulièrement forte et articulée $e^{9}$.» L'expression de la distinction madawaskayenne a un impact sur l'ensemble de la collectivité acadienne. Ainsi Desjardins souligne-t-il «le peu d'appartenance acadien dans la région du Nord-Ouest», ce qui influe négativement sur le degré de cohésion nationale en Acadie. De même, Johnson relève le fait que l'affirmation de l'identité brayonne a amené des organismes sectoriels à passer du «label "acadien" au label "francophone" 10 ». Tel fut le cas, par exemple, de l'Association des instituteurs acadiens, qui est devenue l'Association des enseignants francophones du Nouveau-Brunswick en 1967 à la demande des enseignants du Madawaska, et du Conseil économique acadien du Nouveau-Brunswick, qui a laissé tomber en 1982 le vocable "acadien" afin d'augmenter ses effectifs dans le nord-ouest de la province ${ }^{11}$. Bref, le débat identitaire madawaskayen a des enjeux qui dépassent les limites de la République du Madawaska et qui interpellent l'Acadie dans son ensemble.

Pour bien comprendre les tenants et les aboutissants du débat sur l'identité au Madawaska et ses liens avec l'identité acadienne, plusieurs dimensions demeurent toutefois mal connues, dont celle des fondements historiques du sentiment d'appartenance régionale. Quelques mémoires de maîtrise jettent un peu de lumière sur le sujet. Le travail le plus récent est celui de Nadine Belzile, qui s'est intéressée, dans une perspective anthropologique, à l'affirmation de l'identité brayonne entre 1960 et $1999^{12}$. Son enquête, repo-

9. Luc Desjardins, op. cit., 46; Marc Johnson, Les stratégies de l'acadianité : analyse sociohistorique du rôle de la presse dans la formation de l'identité acadienne, thèse de doctorat (sociologie), Université de Bordeaux II, 1991, 266. Voir aussi l'étude de Gisèle Rhéaume, Conscience territoriale et/ou communautaire et sentiment d'appartenance des populations d'une zone frontalière : étude de perceptions et de comportements - le cas du Madawaska (Nouveau-Brunswick) et de St. John Valley (Aroostook-Nord, Maine), thèse de maîtrise (géographie), Université d'Ottawa, 1977, en particulier 215 et 318, où l'auteure relève le fait que le «sentiment d'appartenance ressenti par les Madawaskayens pour leur milieu propre $[\ldots]$ est fort et relativement dynamique».

10. Luc Desjardins, op. cit., 251, et Marc Johnson, op. cit., 267.

11. Maurice Basque, De Marc Lescarbot à l'AEFNB : histoire de la profession enseignante acadienne au Nouveau-Brunswick (Edmundston, Marévie, 1994), 120; et Wendy Johnston, Sur la lancée de l'an 2000 : 20 ans au coeur de l'économie, 1979-1999 (Moncton, Éditions d'Acadie, 1999), 60-66.

12. Nadine Belzile, Solitudes acadiennes : l'étude de l'identité brayonne basée sur une analyse du courant culturel circulant au Madawaska dans la presse locale entre 1960 et 1999, mémoire de maîtrise (anthropologie), Université Laval, 2000. 
sant sur un échantillon d'articles de l'hebdomadaire local Le Madawaska, lui permet de constater que le phénomène de l'identité brayonne est «bien réel» et "pas aussi marginal que certains voudraient le croire», mais que celle-ci n'est pas pour autant exclusive. On peut être à la fois brayon, acadien ou francophone, selon la stratégie identitaire qui s'impose à la lumière des événements ${ }^{13}$. L'étude de Belzile laisse l'historien sur sa faim; la minceur de son assise documentaire et son caractère échantillonné empêchent toute forme de réflexion fondée sur le caractère évolutif de la construction de l'identité régionale. Deux autres mémoires de maîtrise mettent à profit une véritable perspective historique, soit celui de Georgette Desjardins, sur l'idéologie du journal Le Madawaska entre 1925 et 1927, et celui de Claire Sirois, sur le même journal mais pour la période allant de 1935 à $1945^{14}$. L'auteure du premier mémoire constate qu'au milieu des années 1920 le propriétaire de cet hebdomadaire régional met sa plume au service de la cause acadienne et affiche clairement l'appartenance du Madawaska à l'Acadie, sans jamais toutefois négliger d'insister sur son ancrage régional. Quant à l'auteure du second mémoire, elle arrive à des conclusions similaires pour la période subséquente. Ces deux études ont toutefois le défaut de leur qualité; exhaustives, elles ne portent que sur de brèves périodes. Elles ne permettent donc pas de voir l'évolution du sentiment à l'égard de l'acadianité et des manifestations de l'identité madawaskayenne. Or les identités, qu'elles soient nationales, ethniques ou régionales, n'existent pas en soi; elles sont le fruit d'une construction historique ${ }^{15}$. Si aujourd'hui les Québécois de langue française se disent québécois tout court et non plus canadiens ou canadiens-français, c'est que la perception qu'ils ont d'eux-mêmes a changé au fil des années 1960, au moment où la Révolution tranquille bouleversait les données fondamentales de l'expérience historique canadienne-française ${ }^{16}$. L'identité

13. Ibid., 121, 124.

14. Georgette Desjardins, L'idéologie acadienne du journal Le Madawaska, 1925-1927, mémoire de maîtrise (histoire), Université de Moncton, 1978; et Claire Sirois, Le Madawaska «trait d'union entre l'Acadie et la province qui se souvient " : étude du milieu madawaskayen et de l'idéologie du journal régional, Le Madawaska, de la période 1935-1945, mémoire de maîtrise (histoire), Université d’Ottawa, [1974].

15. Voir à ce sujet l'ouvrage d'Anne-Marie Thiesse, op. cit., qui en fournit un excellent témoignage, ainsi que l'article de René Verrette sur le cas de la Mauricie : René Verrette, «Le régionalisme mauricien des années 1930 ", Revue d'histoire de l'Amérique française, 47,1 (été 1993) : 27-52. Plus généralement, voir l'ouvrage coordonné par Jean-Claude Ruano-Borbalan, dir., L'identitél'individu, le groupe, la société (Auxerre, Sciences humaines éditions, 1998).

16. Voir le récit de cette mutation dans Yves Frenette, Brève histoire des Canadiens français (Montréal, Boréal, 1998) et dans Fernand Harvey, «Le Québec et le Canada français : l'histoire d'une déchirure ", dans Simon Langlois, dir., Identité et cultures nationales (Sainte-Foy, Les Presses de l’Université Laval, 1995), 49-64. 
"québécoise» est historiquement construite, et sa définition même évolue continuellement, à la lumière notamment des débats sur la citoyenneté et le nationalisme civique qui agitent le Québec au tournant du millénaire ${ }^{17}$.

L’identité des gens du nord-ouest du Nouveau-Brunswick est tout autant construite et toujours en chantier. L'utilisation du terme "Brayon" pour identifier les habitants de la région est en effet plutôt récente. Du moins, celui-ci ne figure pas dans l'histoire de la région publiée par l'abbé Thomas Albert en $1920^{18}$. De même, si les expressions «Républicain» et «Brayon» sont fréquemment utilisées pour nommer les habitants de la région dans les années 1980, elles sont décriées par certains comme étant inexactes historiquement ou même, pour ce qui est de la seconde, péjorative ${ }^{19}$. Dans un sondage réalisé par la Société historique du Madawaska en 1978, 65,5\% des répondants se disent certes «Républicains» et 62,7\% «Brayons», mais $41,8 \%$ des personnes interrogées se déclarent aussi «Acadiens ${ }^{20}$ ». Dans les années 1990, au moment où l'identité brayonne s'affiche souvent triomphante, des Madawaskayens œuvrent avec acharnement à ancrer l'idée que le Brayon est également acadien, et que le Madawaska forme l'"Acadie des terres et forêts ${ }^{21}$ ». Bref, le Madawaska, enclavé dans ses limites politiques, linguistiques et naturelles, est la république de l'ambiguïté identitaire et de la multi-appartenance.

Le présent article traite de la question de l'identité au Madawaska, dans le nord-ouest du Nouveau-Brunswick, au $\mathrm{xx}^{\mathrm{e}}$ siècle. Il se penche sur la

17. Pour un aperçu des débats sur le sujet, voir Michel Venne, dir., Penser la nation québécoise (Montréal, Québec/Amérique, 2000). La réflexion de l'historien Gérard Bouchard mérite d'être prise en compte. Voir en particulier Gérard Bouchard, Dialogue sur les pays neufs, propos recueillis par Michel Lacombe (Montréal, Boréal, 1999).

18. Adrien Bérubé, "Thomas Albert et la République du Madawaska», Revue de la Société historique du Madawaska, VIII,1 (janvier-mars 1980) : 12.

19. Voir parmi d'autres Guy R. Michaud, «Des Madawaskayens», Le Soleil, Québec, $1^{\text {er }}$ septembre 1985. On ne s'entend pas sur l'origine du gentilé «Brayon». Certains l'associent au fait de broyer le lin, une activité courante dans la région au $\mathrm{XIx}^{\mathrm{e}}$ siècle, ou au nom de l'instrument utilisé, la "braye». Pour d'autres, par contre, le mot est péjoratif; il serait synonyme de "guénilloux». Voir Guy R. Michaud, "Un mot étrange et étranger», Revue de la Société historique du Madawaska, X,1-2 (janvier-juin 1982) : 4-7. Dans le vocabulaire acadien traditionnel, brayon veut dire torchon, guenille, haillon, loque. Celui qui «braye» est plutôt appelé «brayeux». Pascal Poirier, Le glossaire acadien, édition établie par Pierre M. Gérin (Moncton, Éditions d'Acadie/Centre d'études acadiennes, 1995 [1993]), 79 et 80. Sur la popularité des expressions "républicain» et «brayon", voir Don Cayo, loc. cit. et Will Ferguson, loc. cit.

20. André Leclerc et al., "Connais-toi toi-même : rapport d'un sondage sur l'identité et le sentiment d'appartenance de la population du Madawaska, du Restigouche et du Victoria ", Revue de la Société historique du Madawaska, VII,2-3 (avril—septembre 1979) : 4-139, particulièrement 21.

21. Zoël Dionne, «Invitation au compromis», Le Madawaska, Edmundston, 16 août 2000. 
construction de l'identité de cette région fortement francophone, mais isolée des autres régions de langue française de la province, et sur la relation qui s'établit entre l'identité régionale et l'identité acadienne. Il vise à mettre au jour les représentations identitaires qui prennent forme au Madawaska au cours de la période étudiée, en s'appuyant essentiellement sur l'analyse d'un corpus constitué d'éditoriaux et de lettres à la rédaction publiés dans le seul journal local, l'hebdomadaire Le Madawaska, de sa fondation en 1913 jusqu'à la tenue de la première Foire brayonne en $1979^{22}$. Certes, le journal n'incarne pas à lui seul toute la problématique identitaire régionale; on ne peut saisir dans ses pages toutes les dimensions du sentiment d'appartenance régional. Pourtant, à travers ses éditoriaux, les lettres à la rédaction qu'il choisit de publier, la part qu'il fait aux pratiques culturelles dans ses colonnes, Le Madawaska constitue un lieu privilégié de déploiement des stratégies identitaires régionales. Dans la mesure où elles pouvaient l'être, les données recueillies dans le journal ont été par ailleurs mises en lien avec diverses manifestations porteuses d'identité, telles les célébrations de l'anniversaire de l'arrivée des premiers colons ou de la fondation du comté, afin d'avoir un aperçu plus global de l'évolution des représentations identitaires régionales ${ }^{23}$. De prime abord, celles-ci se révèlent diverses et changeantes au fil du siècle. Promotion des liens du Madawaska à l'Acadie, mise en valeur des traits régionaux distinctifs, affirmation de la multi-appartenance, les représentations identitaires régionales se recomposent au fil du siècle, au fur et à mesure que l'environnement externe se modifie.

\section{I - LE MADAWASKA EST UNE TERRE ACADIENNE}

Vu de l'extérieur, le Madawaska offre au début du $\mathrm{xx}^{\mathrm{e}}$ siècle l'image d'une région isolée, sans liens soutenus avec les autres régions francophones. Ainsi, le docteur A. M. Sormany, originaire de Lamèque, dans la Péninsule acadienne, connaît peu de choses de la région avant d'y venir pour la première fois en 1908, au moment de la convention acadienne de Saint-Basile. «Pour nous qui habitions dans la partie orientale de la province, confiera Sormany

22. Un dépouillement exhaustif du journal entre 1913 et 1979 a permis de constituer un corpus formé de 94 articles, soit 58 éditoriaux et 26 lettres à la rédaction. Si une majorité de textes n'offrent qu'une prise de vue sur le lexique identitaire employé, les autres livrent une réflexion plus poussée sur le sujet.

23. Sur ce plan, la documentation disponible est très pauvre. Ainsi, les commémorations et les autres activités populaires porteuses d'identité ont été nombreuses au Madawaska, mais les écrits qu’elles ont générés et les discours qui y ont été prononcés n’ont pas trouvé la voie des dépôts d'archives. Les comptes rendus publiés dans le journal ont été mis à profit, faute de mieux. 
dans les années 1960, à cause des voies de communications très pauvres, presque nulles, le Madawaska était un endroit mystérieux, inconnu, un peu légendaire, situé, bien proche, à l'autre bout du monde ${ }^{24}$.» Niché à la rencontre des frontières du Québec et du Maine, coupé du reste du NouveauBrunswick francophone par les vastes étendues boisées du Restigouche, le Madawaska est effectivement isolé du point de vue géographique, les distances séparant le chef-lieu du comté, Edmundston, des autres foyers francophones du Nouveau-Brunswick se chiffrant en centaines de kilomètres. Il l'est également du point de vue culturel. Ainsi, la région n'est pas systématiquement représentée aux grandes conventions nationales de la fin du XIx ${ }^{\mathrm{e}}$ siècle et du début du siècle suivant, sauf, bien entendu, à celle qui se tient à Saint-Basile en $1908^{25}$. Le grand journal hebdomadaire acadien, L'Évangéline, ne compte que 142 abonnés au Madawaska en 1921, soit le même nombre qu'au Massachusetts ${ }^{26}$. Ses enfants fréquentent autant sinon plus le collège classique québécois de Sainte-Anne-de-la-Pocatière que les établissements acadiens de Bathurst et de Saint-Joseph de Memramcook ${ }^{27}$.

Outre son isolement géographique, le Madawaska se distingue également des autres populations francophones du Nouveau-Brunswick par le poids démographique de sa population d'origine canadienne-française. Si les Acadiens provenant de la région de Sainte-Anne-des-Pays-Bas (l'actuel Fredericton) ont été en 1785 les premiers Blancs à occuper le territoire, le mouvement de peuplement acadien ne dure que quelques années. Il est relayé à partir de la fin du siècle par l'arrivée de nombreuses familles venues du Bas-Saint-Laurent, à la recherche de terres fertiles pour prospérer et pour établir leurs enfants ${ }^{28}$. Même si le profil démographique de la région se complexifie grâce à l'apport d'autres groupes ethniques, notamment d'individus d'origine écossaise, irlandaise et anglaise, elle conserve son caractère largement francophone. Ainsi, au début $\mathrm{du} \mathrm{xx}^{\mathrm{e}}$ siècle, la population du Madawaska, qui s'établit à un peu plus de 12000 personnes, est à environ

24. Centre d'études acadiennes, Fonds A. M. Sormany, 25-5-5, A. M. Sormany, «Souvenirs d'un jeune âge", [1964].

25. Elle est ainsi absente des conventions de 1884 à Miscouche et de 1890 à Pointe-de-l'Église. Voir Georges Sirois, «La participation des Madawaskayens aux conventions nationales (18801908)", Le Brayon [Revue de la Société historique du Madawaska], IV,1 (janvier 1976) : 22-28.

26. Gérard Beaulieu, «L’Évangéline limitée, propriétaire du journal, 1910-1944 », dans Gérard Beaulieu, dir., L'Évangéline, 1887-1982 : entre l'élite et le peuple (Moncton, Éditions d'Acadie/Chaire d'études acadiennes, 1997), 67. Le journal compte alors un total de 3800 abonnés; 3,7\% proviennent du Madawaska.

27. Jacques Paul Couturier, op. cit., 16.

28. Thomas Albert, op. cit., 129-137. 
$94 \%$ d'origine acadienne ou canadienne-française ${ }^{29}$. L'interpénétration des influences démographiques acadienne et canadienne-française est rapidement vue comme un élément distinguant la population de la région de celle de l'Acadie et du Québec. Lors d'une visite pastorale effectuée en 1812, l'évêque de Québec, $\mathrm{M}^{\mathrm{gr}}$ Joseph-Octave Plessis, notait que les «habitans du Madawaska étant un composé des rebuts de l'Acadie et du Canada, forment une peuplade mal unie, indocile, peu disposée à prendre les bonnes impressions qu'un pasteur s'efforce de leur donner ${ }^{30}$ ». Plusieurs décennies plus tard, un correspondant du Moniteur acadien, publié à Shédiac, évoque à l'aube de la première convention nationale acadienne, en 1881, le fait que "les colons du Madawaska, presque sans aucun contact avec les nationalités étrangères ont, par cela même, développé une indépendance assurée $e^{31}$ ». L'auteur utilise aussi le concept de "république» pour décrire la région, qui semble devenue bien docile, compte tenu de la description qu'en faisait Plessis au début du siècle. «Le Madawaska au fond de ses forêts et de ses montagnes, est $[. .$.$] comme une petite république sagement organisée.»$ Pour certains, les traits distinctifs de la population madawaskayenne finissent par donner naissance à une véritable identité distincte, comme le constate Calixte Savoie, un jeune Acadien qui débarque pour la première fois à Edmundston en 1917. «À peine étais-je rendu à Edmundston, écrit-il dans ses Mémoires, que j'appris que les gens n'aimaient pas qu'on les appelle Acadiens et encore moins Canadiens-Français (sic), et cela se comprend. [...] Les gens étaient soit d'ascendance acadienne, soit d'ascendance canadienne-française ou franco-américaine. C'était une conglomération de différents éléments et la seule appellation acceptable à tous était bien Madawaskaiens ${ }^{32}$."

Les impressions du jeune Savoie trouvent écho dans les pages de l'Histoire du Madawaska, une synthèse d'histoire régionale publiée en 1920. Son auteur, l'abbé Thomas Albert, un fils du pays, y brosse les grands traits de

29. Michel Thériault, «Rapport sur l'état de la question madawaskayenne», Revue de la Société historique du Madawaska, XXII,4 (octobre-décembre 1994) : 15-16.

30. "Journal des visites pastorales en Acadie de $\mathrm{M}^{\mathrm{gr}}$ Joseph-Octave Plessis 1811, 1812, 1815 », Les Cahiers, Société historique acadienne, XI,1-2-3 (1980) : 125. Plessis n'a pas changé d'avis en 1815 : «On pourrait même assurer, jusqu’à un certain point, que cette paroisse est composée des Rebuts et de l'écume du Canada et de l'Acadie», ibid., 225.

31. Moniteur acadien, Shédiac, 7 juillet 1881. Une quinzaine d'années plus tard, une correspondance signée du même pseudonyme rappelle aux lecteurs du journal «que la grande majorité du Madawaska n'est pas essentiellement acadienne, mais que cette partie du Nouveau-Brunswick compte aussi un grand nombre de Canadiens", ibid., 5 mars 1895.

32. Calixte Savoie, Mémoires d'un nationaliste acadien (Moncton, Éditions d'Acadie, 1979), 45. 
l'histoire du "pays» du Madawaska où Acadiens et Canadiens français se sont rencontrés pour constituer un "type» distinct, le "vrai type madawaskayen, breton et normand, à la fois entêté et roublard, honnête et gai, actif et intelligent, généreux et plein d'initiative, hospitalier mais impénétrable, particulariste sans exclusion, qui par suite de sa longue séparation, à son tour, des groupes acadiens et canadiens dont il a tiré son origine, nourrit pour eux une égale et sympathique indifférence ${ }^{33}$.» "Oublié» par les Acadiens «authentiques» et "négligé» par les Canadiens français, ce "peuple» a appris, selon l'auteur, à se suffire à lui-même, sans trop avoir à se soucier de se rapprocher des autres populations francophones. Ainsi, le Madawaskayen est citoyen de son "pays», le Madawaska ${ }^{34}$. La construction de l'identité madawaskayenne passe, selon Albert, par la fusion de deux «races» dans le creuset de l'isolement géographique et de l'indifférence culturelle des populations lui ayant donné naissance.

C'est donc dans un contexte identitaire qui semble passablement marqué que le journal Le Madawaska est publié pour la première fois à Edmundston en 1913. Dès le départ, le journal devient le lieu où prend forme un projet identitaire, celui de rattacher les Madawaskayens au rameau acadien. Sans nier le fait que les gens de la région possèdent des caractéristiques qui les distinguent, Le Madawaska fera en effet la promotion de l'Acadie en terre du Madawaska. Le discours identitaire du journal est clair, même s'il n'est pas énoncé explicitement sous forme de programme, comme l'atteste le sous-titre qui orne sa page couverture : «Journal hebdomadaire en Acadie ${ }^{35}$ ». De même, dans les éditoriaux traitant directement ou indirectement de la question identitaire, l'acadianité de la région est affirmée à travers le recours constant à l'épithète "acadien » quand il s'agit de parler du "peuple», de la «race» ou de la "nation» des franco-catholiques du Nouveau-Brunswick ${ }^{36}$. À d'autres occasions, c'est le territoire régional qui est rattaché à l'Acadie, comme le fait l'éditorialiste D’Erlanges en janvier 1914 en parlant de la région madawaskayenne comme de "cette partie-ci de l'Acadie ${ }^{37}$ ». À d'autres moments encore, c'est dans le contexte de la fête de l'Assomption, qui est aussi la fête

33. Thomas Albert, op. cit., 100.

34. Ibid., 101.

35. Le Madawaska, 27 novembre 1913. Voir Alexandre Savoie, Un demi-siècle d'histoire acadienne ([Edmundston], chez l'auteur, 1976), 65-72. Celui-ci est remplacé en 1934 par la mention «Hebdomadaire en Acadie».

36. Ce que constate également Georgette Desjardins, op. cit., 12-13.

37. Le Madawaska, 29 janvier 1914. 
des Acadiens, qu'un éditorial rappelle aux lecteurs du journal qu'il s'agit là de "notre fête nationale ${ }^{38}$ ».

Ces expressions de l'appartenance du Madawaska à l'Acadie s'expliquent en bonne partie durant les premières années d'existence du journal par l'identité de ses propriétaires, qui en sont aussi les rédacteurs, soit l'avocat Maximilien D. Cormier et le docteur A. M. Sormany. Les deux hommes sont, de l'aveu de Cormier, "des Acadiens d'en bas», de ceux qui «font des efforts partout où ils se trouvent pour le développement de la race acadienne $\mathrm{e}^{39}$ ». En effet, Cormier est né à Barachois, dans le sud-est du Nouveau-Brunswick. Il est établi au Madawaska depuis 1902. Sormany, pour sa part, n'est pas précisément «d'en bas», mais de la Péninsule acadienne. Né à Lamèque, il s'installe à Edmundston en 1910. Ces deux individus vont littéralement tenir le journal à bout de bras jusqu'en 1922, en y consacrant temps et argent, tout en s'engageant dans diverses luttes sur la scène locale et provinciale pour la promotion de la langue française et pour l'avancement de la cause acadienne ${ }^{40}$. Sormany sera particulièrement actif dans les milieux nationalistes acadiens, occupant ainsi la présidence de la Société mutuelle l'Assomption et celle de l'Association acadienne d'éducation ${ }^{41}$.

Les deux fondateurs, accaparés par leurs activités professionnelles respectives, vendent toutefois leur journal en 1922 à de nouveaux propriétaires, Athanase-J. Leblanc et Alphonse Chiasson, qui s'en départissent quelques mois plus tard au profit de J. Gaspard Boucher ${ }^{42}$. Ce dernier, établi dans la région depuis 1920, est un jeune agronome originaire de SaintAlexandre, au Kamouraska, mais qui a fait une partie de ses études à l'Université Saint-Joseph de Memramcook. Malgré les origines québécoises du nouveau propriétaire, le journal continue de véhiculer un fort sentiment d'appartenance à l'Acadie. Boucher se rallie entièrement à la cause acadienne en faisant sienne une définition de l'identité qui met l'accent non pas sur l'ascendance, mais plutôt sur le lieu de résidence. Pour Boucher,

38. Ibid., 19 août 1915.

39. Maximilien D. Cormier à Placide Gaudet, 8 décembre 1913, cité par Alexandre Savoie, op. cit., 65 .

40. Sormany brosse un compte rendu de la fondation du journal et de ses premières années d'existence dans un texte rédigé en 1951. Voir Centre d'études acadiennes, Fonds A. M. Sormany, 25.5.4, «Le Madawaska », [juin 1951].

41. Voir Ibid. Cormier fut pour sa part actif en politique; il présida également la succursale locale de la Société l'Assomption. Voir Le Madawaska, 19 janvier 1933 et 21 octobre 1970.

42. Alexandre Savoie, op. cit., 71. Voir aussi Alexandre Savoie, «Biographie de J. Gaspard Boucher», Revue de la Société historique du Madawaska, XII,1-2 (janvier-juin 1984) : 4-71. 
est acadien toute personne d'origine française qui réside dans les Maritimes. C'est notamment le point de vue qu'il formule au moment d'inviter ses lecteurs à célébrer la fête nationale acadienne en 1925. "C'est la fête nationale des Acadiens, écrit-il, notre fête à nous Français du Madawaska comme de toutes les provinces maritimes ${ }^{43}$.» Une telle définition vient élargir la palette des définitions de l'acadianité. Elle s'oppose notamment à une autre définition, inspirée par l'histoire et qui met l'accent sur l'ascendance. Pour un bon nombre de contemporains, en effet, être acadien, c'est être le descendant d'un colon établi dans l'ancienne Acadie ou d'un déporté de $1755^{44}$. La position éditoriale du journal face à la définition de l'identité acadienne se prolonge jusque dans les années 1940, même après l'entrée en politique de Boucher sous la bannière libérale en 1935. Ainsi l'éditorialiste Lucien Fortin souligne-t-il en 1943 que «le peuple acadien, [...] au sens large du mot,_- il est bon de le rappeler parfois - ne comprend pas seulement les descendants des déportés de 1755, mais aussi toute la population de langue française habitant les Maritimes ${ }^{45}$ ». C'est donc une conception de l'identité acadienne qui marie origine ethnique française et lieu de résidence qui est promue par Boucher et son journal et c'est elle qui lui permet de proposer aux habitants du Madawaska, dont une partie est d'origine canadienne-française, de rallier la famille acadienne.

Le sentiment d'appartenance à l'Acadie, promu par le journal Le Madawaska, repose en bonne partie sur le fait que les éléments servant à définir l'acadianité en cette première moitié $\mathrm{du} \mathrm{xx}^{\mathrm{e}}$ siècle sont facilement transposables au Madawaska. Le premier élément, la langue, va certainement de soi. La région est très majoritairement francophone, et plusieurs de ses habitants sont directement engagés durant les années 1920 et 1930 dans les luttes pour l'avancement de la cause française au NouveauBrunswick, notamment dans le domaine scolaire. Ainsi les éditoriaux du journal sont-ils émaillés de références au fait qu'il existe une communauté de langue parmi les populations francophones du Nouveau-Brunswick et que la défense de la langue française cimente les destins du Madawaska et

43. Le Madawaska, 20 août 1925.

44. Voir à ce sujet la typologie proposée par le géographe Adrien Bérubé : «De l'Acadie historique à la Nouvelle-Acadie : les grandes perceptions contemporaines de l'Acadie ", dans Jacques Lapointe et André Leclerc, dir., Les Acadiens : état de la recherche (Québec, Conseil de la vie française en Amérique, 1987), 198-228. Pour l'historien Léon Thériault, cette conception «fortement généalogique» de l'acadianité, longtemps dominante et encore présente dans les années 1970, "excluait par exemple les habitants du Madawaska comme étant d'authentiques Acadiens». Léon Thériault, La question du pouvoir en Acadie (Moncton, Éditions d'Acadie, [1982]), 37-38.

45. Le Madawaska, 18 mars 1943. 
de l'Acadie ${ }^{46}$. Le deuxième élément, la religion, va également de soi : le catholicisme est la «religion d'État» au Madawaska, tout comme dans les autres coins de l'Acadie. Cela justifie notamment l'accent qui est placé sur la célébration du 15 août, certes fête nationale acadienne mais aussi, et surtout, fête de l'Assomption. La solidarité religieuse s'exprime notamment dans le contexte de la lutte livrée à l'époque pour ramener la religion à l'école. De toute manière, foi et langue vont de pair. Comme l'explique Gaspard Boucher, le "patriotisme grandit avec le sentiment religieux ${ }^{47}$ ». La vision de Gaspard Boucher et des fondateurs du journal, qui est aussi partagée par d'autres nationalistes de la même génération, est donc celle d'une Acadie unie et solidaire, d'où leur insistance sur l'acadianité du Madawaska. Unité et solidarité nationales sont les gages de la réussite des combats pour l'avancement de la cause catholique et francophone aux Maritimes.

Outre la langue et la religion, un troisième élément unit le Madawaska à l'Acadie dans le discours sur l'identité acadienne déployé dans les pages du journal Le Madawaska dans la première moitié du siècle. C'est l'histoire. Pour les éditorialistes du journal, l'histoire du Madawaska est avant tout celle des descendants de déportés acadiens venus de Sainte-Anne-desPays-Bas en 1785. Ainsi, au moment de célébrer le $150^{\mathrm{e}}$ anniversaire de l'arrivée des premiers colons au Madawaska, Boucher insiste sur le fait que le Madawaska est une fondation acadienne. Étrangement, il passe complètement sous silence le fait que des colons canadiens-français ont rejoint les Acadiens dès les premiers temps de la colonie, et que les deux groupes, outre les Amérindiens, peuvent revendiquer conjointement le titre de bâtisseurs de la région ${ }^{48}$. L’année suivante, un autre éditorial braque lui aussi exclusivement les feux sur l'apport acadien : «Descendants des exilés de 1755, qui, au prix de grandes souffrances et après des luttes héroïques nous ont transmis intactes leur foi et leur langue, nous devons parfaire ce miracle de la survivance acadienne ${ }^{49}$. " L'histoire justifie donc elle-même l'appartenance du Madawaska à l'Acadie. Les Madawaskayens sont de descendance acadienne, c'est tout.

La lecture de l'histoire qui est proposée dans les pages du journal n'est pas propre à son propriétaire; elle est la même que celle qui est véhiculée quand la fête de l'Assomption est célébrée dans la région, que ce soit à l'initiative de la Société mutuelle l'Assomption ou du curé d'une paroisse

46. Voir Claire Sirois, op. cit., 46.

47. Le Madawaska, 25 juillet 1929; voir aussi 10 août 1933.

48. Ibid., 2 août 1934.

49. Ibid., 29 août 1935. 
quelconque, ce qui survient sporadiquement au cours des années 1920 et $1930^{50}$. Elle est aussi identique à celle qui anime les organisateurs des fêtes du $150^{\mathrm{e}}$ anniversaire de la fondation du Madawaska, célébrée un an d'avance, en 1934. Au cours de grandes manifestations organisées à SaintBasile les 14 et 15 août, ceux-ci proposent de commémorer le " $150^{\mathrm{e}}$ anniversaire des premiers Acadiens au Madawaska ${ }^{51}$ ». Jumelées à la convention régionale de la Société mutuelle l'Assomption, les fêtes constituent un temps fort du calendrier religieux et patriotique madawaskayen en 1934, et l'occasion rêvée de contribuer au renforcement du sentiment d'appartenance à l'Acadie. Plusieurs éléments y concourent. Le point d'orgue de la première journée est le dévoilement d'une plaque commémorative «du premier débarquement des Acadiens au Madawaska ", à Saint-David (Maine), un hommage qui leur est rendu par «leurs descendants ${ }^{52}$ ». À SaintBasile, les banquets offerts lors des deux jours sont servis "par des dames et des demoiselles de la paroisse, vêtues du costume d’Évangéline». Les principaux orateurs, lors d'un grand rassemblement réunissant 5000 personnes, s'adressent aux Acadiens en général et à ceux du Madawaska en particulier, sans s'embarrasser de distinctions plus locales. Finalement, pour clore les festivités, les Madawaskayens peuvent revivre les grandes étapes de la déportation de 1755 grâce à une "grande séance dramatique» intitulée «Le drame du peuple acadien». Une cérémonie comme celle-là, hors du commun, tout comme celle organisée au même endroit à l'occasion de la convention nationale acadienne de $1908^{53}$, illustre les efforts d'une bonne partie de l'élite clérico-laïque pour développer le sentiment d'appartenance à l'Acadie, une préoccupation similaire à celle que l'on retrouve dans les pages du journal Le Madawaska à la même époque. "Ces fêtes ont écrit de nouvelles pages dans l'histoire de l'Acadie, rapporte d'ailleurs Gaspard Boucher dans son journal. Ce fut l'apothéose du miracle acadien ${ }^{54} \ldots$ »

Au même moment, les manifestations identitaires régionalistes ou même régionales sont assez rares dans les colonnes éditoriales du journal.

50. Comme le révèle un inventaire des célébrations de l'Assomption relatées dans les pages du journal Le Madawaska durant les années 1910, 1920 et 1930.

51. Voir notamment ibid., 26 juillet 1934, 2 août, 9 août, 16 août et 23 août 1934.

52. [Hommage aux pionniers], 1934, Saint-David (musée acadien), plaque de bronze.

53. Sur la convention de 1908, voir Le Moniteur acadien, 27 août, 3 septembre et 10 septembre 1908. Le journal note : «Nos compatriotes de la Rivière St-Jean, malgré leur isolement, sont restés franchement acadiens. Ils l'ont amplement prouvé, et de toutes les manières, durant les fêtes de la semaine dernière. Le drapeau acadien flottait, dominant partout, non seulement à SaintBasile, mais à Edmundston, le chef-lieu du comté, et ailleurs. », ibid., 27 août 1908.

54. Le Madawaska, 23 août 1934. 
Généralement, les éditoriaux réfèrent aux habitants de la région en utilisant l'expression "gens du Madawaska», comme on le fait dans le tout premier éditorial, ou «élément français» du Madawaska. Très peu d'éditoriaux utilisent le gentilé "madawaskayen», nettement plus marqué, pour identifier les habitants de la région, comme le fait un éditorial publié en 1937, où l'auteur évoque la "psychologie du Madawaskayen» et «l'âme madawaskayenne ${ }^{55}$ ". Plus encore, aucun éditorial publié entre 1910 et 1940 ne contient le mot «brayon», qui sera fort répandu quelques décennies plus tard. Les expressions régionalistes sont plus affirmées dans la rubrique de l'opinion du lecteur. Les quelques lettres traitant de l'identité que celle-ci contient renvoient une image plus fragmentée de l'identité régionale. Ainsi, un correspondant qui signe «Franco-Américain» en 1914 met l'accent sur le fait que le Madawaska est constitué de «Franco-Acadiens», de «Franco-Canadiens» et de «FrancoAméricains ${ }^{56}$ ». De même, l'auteur d'une lettre ouverte publiée en 1917 un soldat madawaskayen qui écrit du front français - se remémore les "doux attraits de nos belles "Breyonnes Madawaskayennes" 57 ". Le terme est sans doute peu populaire, en raison du sens péjoratif qu’il peut avoir; du moins ne sera-t-il pas revu dans les colonnes du journal avant longtemps. Dans les années 1930, quelques correspondants évitent d’utiliser le mot «acadien », préférant parler de "gens de langue française» ou de «Madawaskaïens", au grand dam d'un autre correspondant, qui y voit la manifestation d'un esprit régionaliste ${ }^{58}$. Par ailleurs, une autre manière de désigner le territoire - et, par extension, ses habitants — apparaît pour la première fois dans le journal en 1933. Il s'agit de l'expression «république». Celle-ci n’est pas nouvelle; on la retrouve dans le Moniteur acadien en 1881. Thomas LeBlanc explique que l'on entend souvent parler de la "République Madawaskayenne», non pas au Madawaska, mais dans la partie sud de la province $^{59}$. Cette expression traduit selon lui le fait que les habitants du Madawaska constituent «un groupe de Canadiens fortement uni, un groupe qui a fait de [son] comté une forteresse inexpugnable de la langue française et des saines traditions catholiques». Pour LeBlanc, finalement, l'expression "république» ne sépare aucunement la population madawaskayenne du reste de l'Acadie. "Même si nous habitons cette "république", conclut-il,

55. Ibid., 25 novembre 1937.

56. Ibid., 6 mai 1914.

57. Ibid., 27 septembre 1917. À noter que l'auteur écrit «breyonne», et non «brayonne», comme on l'écrit de nos jours.

58. Ibid., 5 avril 1934, 13 janvier et 27 janvier 1938.

59. Ibid., 5 octobre 1933. 
nous n'en sommes donc pas moins patriotes, pas moins Acadiens que les autres groupes ${ }^{60}$.» Par ces propos, LeBlanc s'inscrit tout droit dans l'effort de construction identitaire mené par le journal dans les années 1910, 1920 et 1930. Dans les décennies qui vont suivre, le journal continue à faire mousser l'appartenance du Madawaska à l'Acadie, mais en cherchant plus ouvertement à ménager les susceptibilités régionales. Le Madawaskayen, ce soi-disant «Républicain», est-il bel et bien un Acadien?

\section{2 - LE MADAWASKAYEN EST-IL UN ACADIEN ?}

Le paysage identitaire madawaskayen change au milieu du siècle. Du moins voit-on apparaittre d'autres affirmations, porteuses d'une plus grande distinction régionale. La plus originale, celle qui traduit le mieux la mouvance qui s'installe dans la construction identitaire au Madawaska est sans contredit la popularisation du concept de République du Madawaska et son institutionnalisation à travers la mise en place de diverses représentations symboliques «officielles». Déjà présent dans les pages du journal à quelques reprises, ce concept refait surface en 1941 sous la plume de Gaspard Boucher. Celui-ci parle de «ce petit coin du sol canadien que l'on appelle le Madawaska, et que l'on qualifie de république, par raison des caractères ethniques de son petit peuple ${ }^{61} »$. Boucher expliquera quelques années plus tard ce qu'il entend par ce terme, soit le fait que la population régionale, bien que très majoritairement francophone et catholique, résulte selon lui d'un mélange d'Acadiens, de Canadiens français, d'Anglais, d'Écossais et d'Irlandais ${ }^{62}$. La popularité du concept de «République du Madawaska » croît à partir de la fin des années 1940, au moment où Gaspard Boucher et d'autres élites le mettent au goût du jour pour servir d'outil de promotion touristique. Boucher et un comparse, le docteur Paul Carmel Laporte, conçoivent des armoiries pour la République et les font enregistrer par le bureau des marques de commerce en 1947. Un premier certificat de citoyenneté honoraire est remis la même année. On lance également en 1955 l’Ordre de la République du Madawaska $^{63}$. Les autorités municipales et celles de la Chambre de commerce accréditent le concept en l'incluant dans le titre de deux publications ${ }^{64}$.

60. Ibid.

61. Ibid., 17 juillet 1941.

62. Le Soleil, 14 août 1951.

63. Robert Pichette, loc. cit., 28-34, particulièrement pages 28 et 31 et, du même auteur, «"Les enseignes de sa tribu" : le drapeau de la République du Madawaska ", Revue de la Société historique du Madawaska, XXII,4 (octobre-décembre 1994) : 35-39.

64. Ibid., note 44 et Chambre de commerce d'Edmundston, Edmundston, Nouveau-Brunswick, chef-lieu de la République du Madawaska (Edmundston, la Chambre, [1949]). 
À l'extérieur de la région, la République gagne en notoriété à la suite de la publication d'articles s'y référant dans le journal Le Soleil en 1951 et dans le magazine Maclean's en $1952^{65}$. La compagnie de téléphone du NouveauBrunswick accole même le nom de Republic à la centrale téléphonique d'Edmundston. Peu à peu, donc, les expressions «République du Madawaska» et «Républicains» s'installent dans le paysage identitaire régional. Pourtant peu suspect jusque-là de vouloir accentuer la différence madawaskayenne au détriment des liens avec l'Acadie, Boucher aura ainsi contribué à mettre au monde une République du Madawaska dont l'impact ne sera pas simplement touristique, mais aussi identitaire ${ }^{66}$. Outre la question de la composition de la population régionale, le concept de République du Madawaska, tel qu'entendu au tournant des années 1940, repose sur l'appropriation par ceux qui s'en réclament d'un certain nombre d'éléments de l'histoire ou de la tradition locale, que ce soit la prétendue boutade d'un vieux colon affichant son esprit d'indépendance par rapport aux autorités en se déclarant «citoyen de la République du Madawaska» ou le statut ambigu du territoire avant la conclusion du traité de Webster-Ashburton en $1842^{67}$. Il est en quelque sorte validé par l'auteur d'un travail de recherche de mâ̂trise portant sur l'histoire et la mentalité régionales, l'abbé C. V. Leclerc, qui conclut que l'imprécision des frontières, l'isolement géographique ainsi que l'esprit d’indépendance et la «mentalité spéciale» de sa population justifient le fait que l'on parle de la République du Madawaska ${ }^{68}$. Dans les années qui

65. Le Soleil, 14 août 1951 et Ian Sclanders, "The Rollicking Republic that Doesn't Exist», Maclean's Magazine, 15 octobre 1952, 20-21, 53-56.

66. Le cheminement de la pensée de Boucher à cet égard est difficile à saisir. Son journal reste engagé du côté de l'Acadie. Lui-même souligne pourtant le caractère original du peuplement du Madawaska. Malheureusement, ses archives personnelles sont muettes sur toute la question de l'identité régionale, et plus particulièrement sur la fabrication de la République du Madawaska. Voir Centre d'études acadiennes, Fonds Gaspard Boucher, 211, 1921-1955.

67. Le Soleil, 14 août 1951; La République du Madawaska (Edmundston, Le Madawaska ltée, s.d.); Robert Pichette, "La République...», loc. cit., 28-34. Plus récemment, ce sont les faits et gestes dans les années 1820 d'un colon d'origine américaine qui servent de justification au concept de République du Madawaska. Selon certains historiens, John Baker a en effet voulu instituer une république sur le territoire contesté de la partie supérieure du fleuve Saint-Jean en 1827, son objectif étant l'annexion du territoire aux États-Unis. Voir Roger Paradis, «John Baker and the Republic of Madawaska : An Episode in the Northwest Boundary Controversy ", Dalhousie Review, 52,1 (1972) : 78-95.

68. Centre de documentation et d'études madawaskayennes, Fonds de la Banque d'information et d'études régionales, 2-4-9, C. V. Leclerc, "Thèse pour la maîtrise ès arts - sujet : la République du Madawaska», 2 mai 1949. Le manuscrit est publié dans un ouvrage au titre évocateur : C. V. Leclerc, Saint-André-de-Madawaska, N.-B., une paroisse dans la "République», 19041954 (Moncton, Imprimerie acadienne [1954]). 
vont suivre, malgré son caractère légendaire et sa fonction touristique d'origine, la République du Madawaska sera fréquemment invoquée pour identifier le territoire du Nord-Ouest du Nouveau-Brunswick et sa population.

Les deux grandes activités de commémoration qui se déroulent à la même époque, soit la célébration du bicentenaire de la déportation des Acadiens en 1955 et les fêtes du $175^{\mathrm{e}}$ anniversaire de la fondation du Madawaska en 1961 (au lieu de 1960, comme cela aurait dû être), illustrent à leur manière l'ambiguiité identitaire qui se manifeste au milieu du siècle. Le bicentenaire de la déportation des Acadiens en 1955 est ainsi souligné au Madawaska par une semaine de "réjouissances ${ }^{69}$ ». Encore une fois, une partie des élites madawaskayennes affichent leur sentiment d'appartenance à l'Acadie et contribuent à la commémoration de l'un des événements fondateurs de l'acadianité : la déportation. À Edmundston, l'événement n'est toutefois pas célébré de manière indépendante, mais au même moment que le cinquantième anniversaire de l'incorporation de la ville. Un grand banquet réunit les élites locales à l'Université Saint-Louis, où s'entremêlent les références au jubilée d'or de la ville et au bicentenaire de la déportation. La population locale peut, pour sa part, assister à un impressionnant défilé de chars allégoriques dans les rues de la ville. Ici encore, les deux intentions commémoratives cohabitent : les chars qui se succèdent évoquent pêlemêle la déportation, l'industrie forestière, Évangéline, la Brayonne, l'arrivée des Acadiens au Madawaska, le service d'aqueduc, etc. ${ }^{70}$. Les festivités culminent lors de la présentation d'un pageant qui se révélera fort couru à SaintBasile. Ce jeu scénique, écrit par l’auteur québécois Laurent Tremblay, met en scène une centaine de figurants appelés à reconstituer divers tableaux de l'histoire régionale, où les éléments des deux commémorations sont également intégrés ${ }^{71}$. De leur côté, les fêtes entourant le $175^{\mathrm{e}}$ anniversaire de la fondation du Madawaska proposent une lecture de l'histoire où la dimension acadienne occupe une part plus congrue. Certes, dans un article du journal, le comité des fêtes réitère clairement que les "premiers habitants du Madawaska étaient des descendants des malheureux Acadiens déportés d'Acadie», mais l'accent est essentiellement placé sur la fondation de la colonie comme telle et sur le souvenir des "pionniers fondateurs ${ }^{72}$ ». Ce sont

69. Le Madawaska., 30 juin, 7 juillet et 14 juillet 1955.

70. Ibid., 14 juillet 1955.

71. Sur ce que l'auteur a nommé le Pageant d’Edmundston, voir Raymond Pagé, «L'Acadie en jeu ", dans Raoul Boudreau et al., Mélanges Marguerite Maillet (Moncton, Éditions d'Acadie et Chaire d'études acadiennes, 1996), 271-282.

72. Le Madawaska, 3 août 1961. 
ces mots qui incarnent la raison d'être des célébrations organisées à SaintBasile les 4, 5 et 6 août 1961. Plus encore, le monument commémoratif dévoilé pour l'occasion ne contient que les noms des premiers colons, sans mention du fait qu'ils sont acadiens. À la différence de la plaque posée lors des fêtes du $150^{\mathrm{e}}$ anniversaire, la référence à l'Acadie est donc absente du geste commémoratif. Ce qui est commémoré, ce n'est plus la mémoire des "pionniers acadiens", mais celle des "pionniers du Madawaska», qui sont les «fondateurs de la petite patrie madawaskayenne ${ }^{73}$ ». La référence géographique prend ainsi le pas sur l'histoire pour imposer une autre lecture de l'identité régionale, axée non plus sur sa filiation acadienne, mais sur son ancrage territorial. Au moment de bénir le monument, l'évêque du diocèse, $\mathrm{M}^{\mathrm{gr}} \mathrm{J}$.-Roméo Gagnon, évoquera pour sa part l'unité et la vitalité du peuple du Madawaska ${ }^{74} \ldots$

L’ambiguïté identitaire sous-jacente aux activités de commémoration et des pratiques culturelles des décennies du milieu du siècle trouve éventuellement écho dans les pages du journal Le Madawaska, dont le positionnement identitaire se modifie au tournant des années 1960. Le journal reconnaît ainsi en 1960 que la question de l'identité régionale ne fait pas l'unanimité, et que ce ne sont pas tous les habitants de la région qui acceptent de se déclarer Acadiens. L'éditorialiste Marcel Sormany évoque ainsi les «attitudes diverses » de la population régionale face à la participation à la «vie nationale acadienne». Certains, en raison de l'histoire de la région et des sources de son peuplement, ont selon lui le sentiment d'appartenir au groupe canadien-français au sens large du mot. D’autres sont d'avis que la «république» madawaskayenne traduit bel et bien une réalité profonde. D’autres encore, selon Sormany, mettent l'accent sur l'origine des premiers colons venus s'établir dans la région pour proclamer l'appartenance du Madawaska à l'Acadie. Sormany ne prend pas position pour trancher le débat. Au contraire, il laisse entendre que des identités diverses peuvent coexister; l'idée de base, selon lui, étant que les gens du Madawaska partagent les mêmes problèmes que les Acadiens, d'où la nécessité de travailler avec les "parlants français du reste de la province». "Qu'on se dise Canadien-Français (sic), Madawaskayen tout court ou Acadien, on ne peut manquer de voir que nos problèmes sont les mêmes dans l'ensemble que ceux du reste de l'Acadie ${ }^{75}$.» Ce texte marque un tournant dans le traitement éditorial de la question identitaire dans

73. Monument souvenir [À la mémoire des pionniers du Madawaska], 1961, Saint-Basile (cimetière), béton et plaque de bronze.

74. Le Madawaska, 10 août 1961.

75. Ibid., 28 juillet 1960. 
Le Madawaska; il s'agit là d'une nette reconnaissance de la diversité identitaire madawaskayenne. Il introduit une autre manière de considérer la participation de la région à la vie de la collectivité francophone provinciale. Celle-ci ne repose plus sur la base d'une identité commune, l'identité acadienne, mais sur la base d'une langue commune, le français.

En dépit des efforts de construction identitaire déployés dans ses colonnes et par une partie de l'élite régionale au cours des dernières décennies, Le Madawaska reconnait donc qu'un fort sentiment de distinction anime une partie de la population régionale, une distinction qui s'exprime notamment par un refus de rattachement à l'Acadie. Le fondateur du journal lui-même, le docteur A. M. Sormany, le reconnait lors d'une causerie prononcée en $1951^{76}$. «C'est là une question délicate et difficile à analyser explique-t-il au sujet de l'identité madawaskayenne. La tendance à ne pas se compter comme Acadien est assez répandue chez nous. On a autant de répugnance à se dire Canadien [c'est-à-dire Canadien-Français] (sic). On est Madawaskaïen et ça finit là», constate Sormany. La cause d'un tel sentiment : l'isolement. Peu de choses sont en effet venues rompre l'isolement des régions acadiennes les unes par rapport aux autres. Du point de vue des infrastructures, par exemple, la principale route interprovinciale, qui deviendra la trancanadienne dans les annéees 1950, longe la vallée du fleuve Saint-Jean, et relie Edmundston à Fredericton, Saint-Jean et Moncton. Celles qui permettent d'atteindre les autres communautés francophones demeurent des routes secondaires, qui sont d'ailleurs en mauvais état. Sur le plan culturel, les Madawaskayens ne sont pas en mesure de recevoir le jour même le seul quotidien acadien à vocation provinciale, L'Évangéline. Celui-ci ne compte d'ailleurs qu'environ $2 \%$ de ses abonnés néo-brunswickois. L'arrivée des médias électroniques change initialement peu de choses; les antennes acadiennes de la radio et de la télévision de Radio-Canada, dont les studios sont situés à Moncton, n'émettent pas au Madawaska avant les années $1980^{77}$. Malgré tout, Sormany croit que les choses ont changé au cours des dernières décennies, et que l'on «semble beaucoup plus disposé qu'il y a 20 ou 30 ans à considérer la République comme faisant partie de la population française du Nouveau-Brunswick». Sormany est optimiste. Les voies empruntées dans la construction identitaire dans les années 1960 et 1970, à la fois dans les pages de son journal et dans la société, sont en effet opposées au sentiment qu'il exprime en 1951.

76. Centre d'études acadiennes, Fonds A. M. Sormany, 25.5.4, A. M. Sormany, «La République du Madawaska", [juin 1951].

77. Gérard Beaulieu, op. cit., 101 et Jean-Claude Vernex, op. cit., 305-308. 


\section{3 - LE MADAWASKAYEN EST UN BRAYON, MAIS EST-IL AUSSI UN ACADIEN ?}

À partir du milieu des années 1960, la personnalité du journal Le Madawaska sur le front identitaire change totalement. Tant les éditoriaux que les lettres à la rédaction (qui se font nettement plus nombreuses) renvoient l'image d'une identité circonscrite au territoire régional et exclusive, généralement coupée de liens avec l'acadianité. Ainsi donc, dans presque tous les éditoriaux publiés dans les années 1960 et 1970, l’identité acadienne du Madawaska est contestée et remplacée par de multiples références à l'appartenance madawaskayenne, républicaine ou même, nouveauté, brayonne. Le Madawaska continue donc de contribuer à la construction identitaire régionale, mais dans une optique tout autre que celle des décennies précédentes. Ce changement est en partie attribuable à l'arrivée d'un nouveau groupe d'éditorialistes : Eymard Corbin, Pierrette Verret et Yvette Lagacé. Ces trois journalistes, qui se succèdent à la fin des années 1960 et au début des années 1970 à la barre de la page éditoriale, reflètent et attisent à coup d'effets de plume les tensions entre les communautés francophones néo-brunswickoises. Ainsi, Corbin, qui vient de Grand-Sault, affectionne particulièrement l'expression «République» quand il parle du pays madawaskayen, non seulement pour faire mousser les avantages qu'un tel mot renferme du point de vue touristique, mais aussi pour marquer ce qu'il perçoit comme étant le sentiment d'appartenance régionale. Plus encore, Corbin est le premier à utiliser systématiquement dans les colonnes éditoriales du journal l'expression «brayon», une autre manière de définir plus exclusivement la population régionale, mais qui avait rarement été utilisée publiquement jusque-là. Pour Corbin, le portrait est clair : les Brayons forment un groupe distinct à l'échelle du Nouveau-Brunswick : «Nos mœurs et nos traditions ont des éléments qui leur sont distincts et qu'on ne retrouve pas ailleurs, dans certains cas. Notre parler a un vocabulaire particulier. Il a une histoire magnifique d'endurance, de fierté et, comme tout le reste du monde, d'hospitalité ${ }^{78}$.» Les trois éditorialistes vont même, chacun en leur temps, aller plus loin et remettre en question l'usage du mot "Acadien» pour décrire les francophones du Nouveau-Brunswick. Ce nom, selon Corbin, doit être remplacé : "pensons à un autre mot pour remplacer acadien, qui n'a plus d'éclat au Madawaska ${ }^{79}$ ». Pour Pierrette Verret, le mot «Acadien » est porteur de division parmi les francophones de la province. L'éditorialiste pousse sa réflexion 
jusqu'à remettre en question l'existence même d'une communauté française au Nouveau-Brunswick, qui est, selon elle, «une réalité de théorie et non de fait ${ }^{80}$ ». Quand il s'agit de nommer Madawaskayens et Acadiens, les éditorialistes du journal optent pour des termes neutres : "communauté francophone des Maritimes", "francophonie du Nouveau-Brunswick», "francophones», à l'image du vocabulaire employé parmi d'autres minorités francophones du pays et par les organismes fédéraux qui se chargent de distribuer les retombées de la nouvelle politique fédérale de promotion des minorités linguistiques ${ }^{81}$.

Ce refus de s'identifier à l'Acadie est encore plus fort parmi ceux et celles qui s'expriment dans les colonnes de l'opinion du lecteur, quoique les manifestations régionalistes y apparaissent plus tardivement, se situant surtout dans les années 1970. La grande majorité des auteurs des lettres publiées affichent ainsi haut et fort leur appartenance au Madawaska, à la République et au "peuple» brayon. Seulement quelques-uns reprennent la position affichée par le journal jusqu'au début des années 1960, soit qu’est acadien tout individu de langue française qui réside aux Maritimes, peu importe son origine ethnique, tout en prenant aussi bien soin de réaffirmer leurs racines madawaskayennes et brayonnes. «Je suis moi-même un Brayon pur sang, un Madawaskayen pure laine, écrit l'un d'eux, curé et candidat du Parti acadien dans la région du Restigouche. Je ressens cette teinte originale et cette vocation unique de notre région, mais je suis aussi un Acadien; l'un n'exclut pas l'autre, au contraire, il le complète ${ }^{82}$.» Pourtant, même ceux qui affirment sur ces bases leur appartenance à l'Acadie doivent concéder qu'une telle définition de l'acadianité n'est pas partagée par tous les Acadiens, et que dans les autres régions de la province, l'acadianité est souvent présentée et vécue comme étant fonction de l'ascendant, comme l'écrit l'auteur d'une lettre à la rédaction en 1972 : «Cependant, il n’y a pas qu'au Madawaska qu'on donne au mot un sens exclusif. Nombreux encore aujourd'hui sont les descendants des déportés de 1755 qui, habitant d'autres régions francophones du Nouveau-Brunswick, ne reconnaissent pas comme vivant en Acadie les habitants du Madawaska. C'est cette double négation qui empêche les francophones de se rallier autour du terme

80. Ibid., 19 décembre 1968.

81. Ibid., 6 mars et 19 décembre 1969. Au sujet de l'impact de l'intervention fédérale sur le lexique identitaire des minorités francophones, voir Michel Bock, Comment un peuple oublie son nom : la crise identitaire franco-ontarienne et la presse française de Sudbury (1960-1975) (Sudbury, Prise de parole/Institut franco-ontarien, 2001).

82. Ibid., 12 octobre 1978. 
“acadien" ${ }^{83}$.» Dans un tel contexte, la vision «territoriale» de l'identité acadienne proposée par le journal Le Madawaska se bute donc non seulement à la vigueur de l'identité madawaskayenne, mais aussi à l'image de l'Acadie qui lui est renvoyée par l'Autre, l'Acadien.

La principale célébration commémorative des années 1970 s’inscrit dans la même trajectoire identitaire que celle qui émerge des lettres à la rédaction et des éditoriaux du Madawaska; elle participe tout autant à la construction d'un vigoureux sentiment d'appartenance au territoire madawaskayen. L’année 1973 marque le centième anniversaire de la création du comté de Madawaska. Dans l'album-souvenir publié pour l'occasion, la plupart des textes mettent bien entendu l'accent sur les hauts faits et les particularités de l'histoire régionale ${ }^{84}$. Chose plus étonnante, toutefois, les dignitaires appelés à offrir leurs bons vœux aux Madawaskayens puisent abondamment dans le mythe de la République du Madawaska pour souligner la commémoration de la fondation du comté. Du premier ministre Richard Hatfield jusqu'aux élus locaux, tous ou presque utilisent les mots «République du Madawaska » dans leur message à l'intention des Madawaskayens. De plus, ceux qui soulignent les origines du peuplement régional placent les apports acadien et québécois sur un même plan, ce qui n'était pas le cas lors des autres entreprises de commémoration. Pour l'évêque du diocèse, par exemple, "le "Brayon" est un type bien caractérisé de Canadien, rencontre d'Acadiens de nouveau "expulsés" du sud et de Québécois venus chercher ici les moyens de vivre ${ }^{85}$ ». Au début du $\mathrm{xx}^{\mathrm{e}}$ siècle, le Madawaska commémorait ses origines acadiennes; dans les années 1970, il célèbre sa distinction.

Cette manière de se voir et de se représenter trouve écho au sein de la Société historique régionale, relancée en 1971. Dans sa revue, nommée Le Brayon afin de rendre hommage aux pionniers de la région, les membres valorisent la richesse du patrimoine régional et le caractère distinct de sa population ${ }^{86}$. «Les Madawaska et ses habitants ont un passé assez étonnant et particulier, peut-on lire dans la revue en 1974. C’est sans doute ce passé qui a engendré des individus fort différents des autres habitants du Nouveau-Brunswick : les brayons ${ }^{87}$.» L'expression de la spécificité mada-

83. Ibid., 21 juin 1972.

84. Livre souvenir - Centenaire du Madawaska, 1873-1973 (s.1., s.é, s.d.).

85. Ibid., 6.

86. Voir Le Brayon, 1,2 (printemps 1972) : [2].

87. Cécile Gagnon, «Mot de la présidente», Le Brayon, 2,1 (mai 1974) : 3. 
waskayenne culmine en 1979 lors de la création de la Foire brayonne, un festival d'été se voulant une grande fête populaire et un événement touristique majeur sur la scène provinciale. Dès le départ, le nom choisi représente un marqueur identitaire très fort. Le contenu de l'événement, les discours qui y sont prononcés lors des cérémonies d'ouverture et de fermeture, le matériel publicitaire préparé pour mousser la fête, tout en fait contribue à renforcer le sentiment d'appartenance au Madawaska et à la République, ainsi que le caractère distinct de sa population ${ }^{88}$. Le drapeau de la République, dessiné par Robert Pichette et adopté par le conseil municipal d'Edmundston en 1965, est bien en évidence. Inspiré de l'étendard hissé par John Baker durant les troubles de la frontière au $\mathrm{xIX}^{\mathrm{e}}$ siècle, le drapeau est composé d'un aigle et de six étoiles posées en demi-cercle sur fond blanc. Sans signification particulière à l'origine, le nombre d'étoiles est peu à peu associé à une notion erronée sur le plan historique, celle des «six peuples fondateurs» du Madawaska, soit les Acadiens, les Canadiens français, les Écossais, les Irlandais, les Anglais et les Autochtones, ayant donné naissance à un nouveau groupe, les Brayons ${ }^{89}$. Ici donc encore, l'histoire est mise à profit dans la construction identitaire. Il s'agit d'un passé en quelque sorte fictif, tout comme celui de la République du Madawaska, mais reçu par plusieurs comme étant réel ${ }^{90}$. Il devient un ingrédient actif dans la construction de la différence madawaskayenne et de l'identité des Madawaskayens et des Madawaskayennes.

Cette thématique des six peuples fondateurs, qui relègue nettement au second plan l'explication des origines acadiennes, pourtant bien en vue dans la première moitié du siècle, est omniprésente dans le cérémonial de la Foire brayonne. Ainsi, lors de la première cérémonie d'ouverture, six couples, vêtus de manière à représenter les six peuples fondateurs, présentent le drapeau de la République à la foule rassemblée. "Aujourd'hui, à l'occasion de l'ouverture de la première foire brayonne, explique le maître des cérémonies, nous voulons nous souvenir de nos devanciers, et rappeler la contribution de chaque peuple au développement d'une mentalité

88. Le Madawaska, 25 juillet, $1^{\text {er }}$ août et 9 août 1979.

89. Sur la question des peuples fondateurs et du nombre d'étoiles, voir Michel Thériault, loc. cit., 5-6, et Robert Pichette, "Les enseignes de sa tribu" ", loc. cit., 38. Signe des temps, les Autochtones ont dorénavant le statut de "peuple fondateur»; ils ne figuraient toutefois pas dans l'énumération proposée par Boucher en 1951. Voir Le Soleil, 14 août 1951.

90. Comme l'explique Joseph-Yvon Thériault : «Peu importe en fait le caractère fictif ou réel des mythes fondateurs, l'important c'est qu'ils donnent une cohésion au groupe et qu'ils mobilisent son action. » Joseph-Yvon Thériault, "Naissance, déploiement et crise de l'idéologie nationale acadienne», dans Simon Langlois, dir., op. cit., 73-74. 
typiquement madawaskayenne, faite de bonne humeur, du sens de l'accueil et de l'hospitalité ${ }^{91}$.» Et tout comme lors des commémorations de 1934 et de 1961, la Foire brayonne va jeter les assises d'une nouvelle mémoire : la réalisation entre 1986 et 1991 de six statues géantes, sculptées à la scie à chaîne par un artisan local, et représentant les «six peuples fondateurs» du Madawaska $^{92}$. Ceux dont la mémoire a été honorée durant plusieurs décennies, les pionniers acadiens de 1785, sont dorénavant relégués au rang de "peuple fondateur», au même titre que les Écossais, les Anglais et les Irlandais. Ironiquement, c'est en bonne partie en langue anglaise, du côté du Madawaska américain, que la mémoire de la fondation acadienne du territoire est entretenue, à travers un festival acadien, tenu annuellement, et l'érection à Saint-David en 1984 d'une immense croix de marbre afin de souligner le bicentenaire de l'arrivée des Acadiens au Madawaska ${ }^{93}$.

Les tangentes identitaires qui se manifestent dans les pages du journal Le Madawaska adoptent donc des formes bien concrètes dans les cérémonies et les témoignages commémoratifs qui sont organisés ou produits dans les années 1960 et 1970. Mais qu'est-ce donc qu’un Madawaskayen ou un Brayon, et quels sont les éléments qui empêchent certains habitants de la région d'être identifiés comme Acadiens? Les fondements de l'identité régionale sont diversifiés, mais reposent essentiellement sur deux éléments : le fait que la région a été peuplée à la fois par des Acadiens et par des Canadiens français, et le fait que ces deux groupes ont longtemps été isolés des autres groupes francophones de la province. À cela s'ajoutent périodiquement d'autres référents, comme la proximité des États-Unis (un argument souvent évoqué au début du $\mathrm{xx}^{\mathrm{e}}$ siècle) ou l'apport de plusieurs groupes ethniques de langue anglaise (ce qui deviendra le concept des «six peuples fondateurs»). «Parce que nous sommes d'origine à la fois acadienne et québécoise, nous nous identifions tout simplement à l'élément francophone du nord-ouest», écrit ainsi Pierrette Verret dans Le Madawaska en $1968^{94}$. Ce récit des origines est repris et approfondi quelques années plus tard par un lecteur fortement engagé dans la promotion du particularisme régional, Oneil Clavet, dans des termes quasi mythiques : "Après la période troublée de la déportation des Acadiens et de la chute du dernier bastion canadien-

91. Le Madawaska, 23 juillet 1980 [texte lu lors de la cérémonie d'ouverture de 1979].

92. Albert Devost, Les six peuples fondateurs, 1986-1991, Edmundston (Hôtel de ville), bois.

93. Le Madawaska, 21 juin et 19 juillet 1978; Acadian Cross Historic Shrine, 1985, Saint-David (Maine), marbre. L'inscription se lit comme suit : «1785-1985 - Acadian Cross Historic Shrine. En l'honneur de nos pionniers. In honor of our Pioneers.»

94. Le Madawaska, 19 décembre 1968. 
français en Amérique [...] deux groupes de colons, l'un venant du sud et l'autre du Québec, s'exilèrent volontairement au "pays des porcs-épics" dans l'espoir d'y retrouver la paix et la liberté. [...] Coupés de toutes communications avec le reste du pays, nos ancêtres vécurent dans un isolement séculaire qui leur a permis de façonner notre personnalité brayonne et de cimenter par les épreuves qu'ils ont partagées, des liens d'une parenté que même les divisions politiques ne pourront jamais effacer ${ }^{95}$." Ainsi donc, le refus de l'acadianité repose sur le fait qu'une nouvelle identité s'est construite à la suite de la rencontre de deux groupes sur une terre isolée, où leur volonté de paix et de liberté a façonné un type distinct du Québécois ou de l'Acadien : le Brayon.

Outre la perception que l'on a de sa propre différence, la représentation que l'on se fait de l'Autre et des traits qui le caractérisent entrent également en jeu dans la construction de l'identité régionale. Plusieurs sont ainsi d'avis que les régions acadiennes sont moins francophones que le Madawaska ne l'est lui-même ou qu'elles ne vivent pas avec la même intensité leur francité. Ce trait est souligné depuis plusieurs années dans le journal, le Madawaska y étant présenté comme la région «la plus française de la province». Est aussi noté le fait que les Acadiens de Moncton, au début des années 1970, semblent peu portés à parler français et à défendre l’usage du français. "Si l'on commençait à parler français à Moncton, écrit Yvette Lagacé, on s'attirerait en tout cas un peu plus de respect de la part des autres francophones de la province ${ }^{96}$. $)$ D'autres traits dits acadiens ont aussi une connotation négative aux yeux de certains Madawaskayens. Ainsi, l'auteur d'une lettre à la rédaction souligne, en 1973, que les Acadiens souffrent d'un complexe d'infériorité et manquent de confiance en eux, tandis que les Brayons sont sûrs d'eux; ils sont «bûcheurs et audacieux ${ }^{97}$ ». Un jugement similaire est formulé dans les pages éditoriales du journal l'année suivante et porte sur la charge identitaire de l'acadianité. «Acadien est devenu au cours des années synonyme de personne sans grande épine dorsale, craintive et lamenteuse», de l'avis de l'éditorialiste Lagacé ${ }^{98}$. Le ton du débat sur l'identité au Madawaska et en Acadie est donc loin d'être serein et généreux au cours des années 1970. Les champions de l'identité brayonne se disent certes ouverts à la collaboration avec les autres francophones de la province dans les dossiers touchant à la défense de la langue

95. Ibid., 2 août 1973. Voir aussi 27 mars 1974 et 22 mars 1978.

96. Ibid., 12 janvier 1972.

97. Ibid., 30 mai 1973.

98. Ibid., $1^{\mathrm{er}}$ mai 1974. 
française, mais à condition que l'on respecte leur caractère distinct et que l'on ne cherche pas à leur imposer une étiquette acadienne dont ils ne veulent pas. Ainsi, à la veille de la création de la Société des Acadiens du Nouveau-Brunswick, dans la foulée du Congrès des francophones, tenu à Fredericton en 1972, l'un des plus ardents défenseurs de l'identité brayonne craint que l'on ne mette au monde " un autre enfant de la thalidomide, un enfant à qui il manquera des membres, parce que les Acadiens, toujours irréductibles, s'acharnent à vouloir imposer le titre d'Acadien à tous les francophones du Nouveau-Brunswick ${ }^{99}$ ".

Ainsi donc, les manifestations identitaires inscrites dans les colonnes du journal Le Madawaska n'ont plus du tout la même forme à partir des années 1960. Tandis que le journal dévie peu d'une étroite association des habitants du Madawaska à l'Acadie dans la première moitié du siècle, à partir des années 1960 et surtout des années 1970, il devient le lieu privilégié de l'expression d'une identité régionale forte et le plus souvent exclusive. Le même phénomène se manifeste dans les champs de la commémoration et des fêtes populaires. Comment expliquer un tel changement? Dans leurs travaux sur la société acadienne contemporaine, les sociologues Marc Johnson et Isabelle McKee-Allain ont mis en évidence ce qu'ils ont appelé le "phénomène de la segmentation de l'identité collective acadienne", qui se caractérise par le relâchement de la cohésion nationale et la montée des manifestations régionalistes et des expressions identitaires régionales ${ }^{100}$. Ce phénomène est vu comme étant le résultat de l'affaiblissement de l'idéologie nationaliste traditionnelle et de l'influence de l'élite cléricolaïque. Cette dernière occupait durant la première moitié $d u x^{e}$ siècle pratiquement tout l'espace idéologique en Acadie; ses membres communiaient à ces mêmes valeurs que sont la ruralité, la préservation de la langue française et l'élargissement de l'aire sociale du catholicisme, en particulier dans le domaine scolaire, ce qui assurait la cohésion et la solidarité du groupe. Ils se côtoyaient au sein des organisations patriotiques et religieuses; ils étaient au coude à coude dans l'Ordre de Jacques-Cartier, communément appelé la "patente». Ils étaient unis par le même souci d'œuvrer pour une cause commune, celle de l'avancement de la francophonie néo-brunswickoise, dans un contexte minoritaire marqué par

99. Ibid., 30 mai 1973. Voir aussi $1^{\text {er }}$ mai 1974.

100. Marc Johnson et Isabelle McKee-Allain, «La société et l’identité de l’Acadie contemporaine», dans Joseph-Yvon Thériault, dir., Francophonies minoritaires : l'état des lieux (Moncton, Éditions d'Acadie/Regroupement des universités de la francophonie hors Québec, 1999), 231. Voir également Patrick Clarke, loc. cit., 331-341. 
l'adversité. Du côté des élites religieuses, dont l'influence était fort grande jusque dans les années 1960, la hiérarchie imposait une communauté de vues qui ne saurait faciliter la dissidence, fût-elle théologique ou régionale. Bref, les structures sociales de la société acadienne lui conféraient une cohérence identitaire qu'elle n'avait peut-être pas en réalité ${ }^{101}$.

Cette piste d'analyse mérite certainement d'être explorée pour comprendre l'évolution des processus de construction identitaire au Madawaska au $\mathrm{xx}^{\mathrm{e}}$ siècle. En effet, tout le discours sur l'acadianité du Madawaska est fortement imprégné d'éléments qui se retrouvent dans l'idéologie clériconationaliste de l'époque, que ce soit le destin providentiel des Acadiens, le rôle salvateur de la religion catholique ou le culte de la mémoire de la présence française en Amérique. Or les référents religieux s'amenuisent au fil des années 1960; la société acadienne vit comme au Québec à l'heure de Vatican II, qui modifie dans son sillage le paysage institutionnel et les comportements personnels : les congrégations religieuses abandonnent le contrôle des collèges et des hôpitaux qu'elles ont fondés, des prêtres et des religieuses constatent qu'ils et elles n'ont plus la vocation, les fidèles désertent les églises ${ }^{102}$. Quant aux référents linguistiques, s'ils persistent, c'est parfois pour adopter une nouvelle forme, celle par exemple de la différence entre un Madawaska bien francophone et une Acadie (surtout celle du SudEst) menacée par l'anglais. Au même moment, le nationalisme acadien est sous tension, en particulier les jeunes qui critiquent sa configuration conservatrice et son peu d'ouverture, et qui vont même jusqu'à temporairement rejeter ses principaux symboles ${ }^{103}$. Si durant longtemps l'idée de la nécessaire solidarité s'est imposée, au détriment même de l'expression des personnalités régionales, le contexte des années 1960 change donc en profondeur la donne et ouvre la voie à l'expression des identités régionales. Celles-ci se

101. Voir notamment Marc Johnson, op. cit., 263-265. La cohérence de vues et d'action est bien démontrée par Alexandre Savoie dans son ouvrage sur l'Ordre de Jacques-Cartier. Voir Alexandre Savoie, Un siècle de revendications scolaires au Nouveau-Brunswick, 1871-1971, 2 : Les commandeurs de l'Ordre à l'ouvre (1934-1939) ([Edmundston], chez l'auteur, 1980).

102. Voir par exemple la thèse d'Isabelle McKee-Allain sur les congrégations religieuses féminines. Isabelle McKee, Rapports ethniques et rapports de sexes en Acadie : les communautés religieuses de femmes et leurs collèges classiques, thèse de doctorat (sociologie), Université de Montréal, 1995. Pour une réflexion sur l'évolution de la problématique identitaire telle qu'elle se pose dans les années 1990, Joseph-Yvon Thériault, "Naissance...», loc. cit., 67-84 et Greg Allain, Isabelle McKee-Allain et J. Yvon Thériault, «La société acadienne : lectures et conjonctures», dans Jean Daigle, dir., L'Acadie des Maritimes : études thématiques des débuts à nos jours (Moncton, Chaire d'études acadiennes, 1993), 378-379.

103. Ibid., 357-364. 
manifestent de différentes manières. Ainsi le Sud-Est se démarque-t-il du Nord-Est et vice versa, chaque ensemble affirmant sa personnalité propre. Les clivages entre régions sont nombreux, mais l'histoire que l'on a en partage, en particulier la déportation, demeure le ciment de l'acadianité hors du Madawaska.

Le champ des rapports sociaux n'est pas le seul qui peut être évoqué pour expliquer l'évolution de l'identité au Madawaska. Un deuxième élément entre en ligne de compte, soit les rivalités régionales qui s'installent, en particulier dans le domaine économique ${ }^{104}$. Jusqu'à l'aube des années 1960, les grandes régions francophones de la province partagent essentiellement le même lot du point de vue socio-économique : sauf dans le secteur de Moncton et dans les poches urbaines de Bathurst et d'Edmundston, ces régions dépendent d'activités liées à l'exploitation des ressources naturelles, soit la pêche, la coupe du bois et l'agriculture. Certes, des rivalités régionales existent dans le domaine économique, mais les enjeux sont somme toute limités. Le principal foyer des tensions interrégionales est probablement le courant de centralisation qui se manifeste dans la sphère institutionnelle et associative en faveur de Moncton, que ce soit à l'Université de Moncton ou au sein de la Société nationale des Acadiens ${ }^{105}$. À plusieurs reprises fait-on ainsi référence dans Le Madawaska au fait que Moncton se considère comme le "nombril de l'Acadie», ou que la ville du Sud-Est constitue le «soi-disant centre de l'Acadie ${ }^{106}$ ». Ce portrait change radicalement dans les années 1960, quand les francophones des Maritimes et les autres Canadiens goûtent peu à peu aux mérites de l'État-providence et d'un État interventionniste, soucieux par le biais de programmes d'infrastructures et de subventions diverses de réduire les effets du sous-développement économique régional ${ }^{107}$. C'est ce qui lance la concurrence entre régions pour obtenir les subventions gouvernementales et les investissements publics. Or le jeu de la rivalité économique s'insinue clairement et régulièrement dans plusieurs des textes où le particularisme madawaskayen se manifeste avec force. Ainsi, l'affirmation de l'identité brayonne dans les pages du journal Le Madawaska en 1968 vient dans la foulée du constat que la visite de quatre Acadiens rendue au président français Charles de Gaulle a peu de retombées pour le

104. Patrick Clarke, loc. cit., 335, 342-343.

105. Sur le cas de l'Université de Moncton, voir Jacques Paul Couturier, op. cit., et Belzile, op. cit., 82-94.

106. Le Madawaska, 11 janvier 1968; voir aussi 12 janvier 1972.

107. Greg Allain, Isabelle McKee-Allain et Joseph-Yvon Thériault, loc. cit., 350-357. Voir aussi Patrick Clarke, loc. cit., 331-341. 
Nord-Ouest ${ }^{108}$. De même, la critique des attitudes du gouvernement provincial dans des dossiers du Nord-Ouest est aussi l'occasion de réaffirmer la distinction locale, que ce soit dans le domaine du tourisme, des routes ou de la gestion du patrimoine historique. "Selon toute apparence, écrit ainsi l'éditorialiste Corbin, il semble y avoir une politique ou une absence de politique pour le nord, et une politique pour le sud ${ }^{109}$.» Oneil Clavet sonne également le clairon sur un thème apparenté dans l'opinion du lecteur : «Madawaskayens, réveillez-vous. La population diminue à vue d'œil, au même rythme que les emplois dans nos industries locales. [Si rien n'est fait], nous deviendrons en peu de temps le parent pauvre d'une province florissante $^{110}$.» De même, l'élite monctonienne est accusée de centralisme et de machiavélisme dans le dossier de l'Université de Moncton, particulièrement dans le contexte de la publication des résultats d'enquêtes majeures comme en 1972 et en $1975^{111}$. Non seulement les anciennes conditions, qui maintenaient intact le vernis de la solidarité, n'existent-elles plus, mais de nouveaux facteurs, telle la concurrence économique, invitent la montée des régionalismes et les expressions identitaires régionales.

\section{CONCLUSION}

Globalement, deux manières de penser l'identité régionale, échelonnées dans le temps, ressortent donc de l'examen de la question identitaire au Madawaska au fil du $\mathrm{xx}^{\mathrm{e}}$ siècle. Une première, que l'on retrouve durant toute la première moitié du siècle dans les éditoriaux et dans quelques lettres à la rédaction publiés dans le journal Le Madawaska, ainsi que dans divers gestes de commémoration organisés dans la région, met l'accent sur la présence d'un sentiment d'appartenance régional fort, mais qui n'est pas exclusif. Le Madawaskayen est aussi un Acadien, à certains moments en raison de son histoire, la plupart du temps à travers une langue et un lieu de résidence partagés. Un Acadien, c'est un francophone - on pourrait dire aussi un catholique — qui habite les Maritimes, ce qui inclut bien entendu la plupart des Madawaskayens. Une deuxième manière de concevoir l'identité régionale s'installe peu à peu dans les pages du Madawaska dans la deuxième partie du siècle, et surtout dans les années 1960, où elle

108. Le Madawaska, 25 janvier 1968. Sur le sujet, voir le livre de Robert Pichette, L'Acadie par bonheur retrouvée : De Gaulle et l'Acadie (Moncton, Éditions d'Acadie, [1994]), notamment 197-208, et Belzile, op. cit., 71-82.

109. Le Madawaska, 21 septembre 1967.

110. Ibid., 27 mars 1974.

111. Par exemple, voir Ibid., 20 juin 1972 et 10 juillet 1975. 
prend bientôt presque toute la place. Elle surgit également à travers la mise en place des représentations symboliques de la République du Madawaska, lors de la célébration du centenaire du comté et durant une grande fête populaire, la Foire brayonne. Cette deuxième forme de représentation identitaire devient bientôt généralement exclusive; elle s'incarne dans les gentilés "Républicain» et «Brayon», ces figures issues de la rencontre de deux peuples et façonnées par un isolement relatif, très attachées à leur "pays». Dans ce contexte, les efforts de construction identitaire de la première partie du siècle, qui visaient à rattacher le Madawaska à l'Acadie, que ce soit dans le journal ou dans les activités de commémoration, semblent avoir été éclipsés par ceux qui prônent le particularisme régional. Décors en trompe-l'œil, œuvre d'une élite lettrée, qui acquiert ainsi un capital symbolique à travers les expressions de solidarité nationale, les tentatives visant à développer le sentiment d'appartenance acadien au Madawaska expriment la coïncidence des intérêts des élites régionales et de celles des autres régions, sous la grande tente du patriotisme et du catholicisme, bien plus peut-être que le réel sentiment d'appartenance de la population régionale dans la première moitié $\mathrm{du} \mathrm{xx}^{\mathrm{e}}$ siècle. Il aurait pu en être autrement, en dépit des éléments qui fondent le particularisme régional. Il ne pouvait peut-être pas en être autrement, dans un contexte où la définition de l'acadianité qui fondait les efforts de construction identitaire - la langue et le lieu de résidence — n'était pas arrimée aux définitions les plus courantes à l'époque de l'identité acadienne, c'est-à-dire liées à la généalogie et à l'histoire. Ce qui ressort des efforts des premières décennies du siècle, à la lumière des développements après la fin de la Seconde Guerre mondiale, c'est le constat d'un échec : l'échec d'une tentative d'acadianisation du Madawaska.

Le Madawaska, petit territoire francophone du nord du NouveauBrunswick, fournit un bel exemple du caractère construit de l'identité d'une région. Tout au cours du $\mathrm{xx}^{\mathrm{e}}$ siècle, l'identité régionale est en chantier, oscillant entre l'adhésion à une vision désenclavée de soi-même, reposant sur une communauté de langue et de territoire, et une fidélité teintée d'exclusivisme aux particularités locales. Dans le premier cas, l'effort de construction identitaire est alimenté par le patriotisme d'une élite cherchant à construire des ponts avec celles des autres régions et à faire progresser la cause nationale. Dans le deuxième cas, il cherche à traduire en une identité propre un sentiment de distinction et de différence par rapport aux collectivités acadiennes et québécoises voisines. Au regard de l'évolution de la situation dans la deuxième moitié du siècle, l'identité acadienne du 
Madawaska, qui est au cœur de l'effort de construction identitaire du début de la période étudiée, peut sembler préfabriquée. Elle l'est peut-être, mais les identités républicaines et brayonnes qui sont issues du deuxième courant de composition identitaire le sont tout autant. Elles expriment peut-être une réalité, mais la manière dont elles l'expriment, à travers le concept de République ou dans la Foire brayonne, tire en bonne partie sa source d'initiatives banalement touristiques. Telle est la raison derrière la création des deux éléments qui contribuent le plus à former l'identité madawaskayenne actuelle. Par ailleurs, tout un ensemble de facteurs, de l'isolement géographique à la composition démographique en passant par l'histoire et la compétition économique, entrent en jeu dans les processus de construction identitaire qui se manifestent au Madawaska au cours du siècle. L'identité est un enjeu, pas simplement culturel, mais aussi économique et politique ; son impact dépasse le cadre régional pour interpeller aussi l'Acadie dans son ensemble. Le cas du Madawaska montre clairement que l'on ne peut pas expliquer l'identité acadienne sans tenir compte des identités régionales. La question identitaire en Acadie se comprend à travers l'identité des régions qui la composent. 